\title{
SOBRECARGA DEL CUIDADOR PRINCIPAL DEL ADULTO MAYOR CON ENFERMEDAD ALZHEIMER
}

\author{
Overload of the caregiver of the adult higher with disease of Alzheimer
}

\section{EPISTEMUS}

ISSN: 2007-8196 (electrónico)

ISSN: 2007-4530 (impresa)

Adriana Elizabeth Aguilar Gutiérrez ${ }^{1}$ Jasmín Jiménez Reyes 2

Alicia Álvarez Aguirre 3 Mercedes Sánchez Perales 4 Marcela Ortega Jiménez 5

Recibido: 14 de octubre de 2016,

Aceptado: 13 de noviembre de 2016

\section{Autor de Correspondencia:}

Dra. Alicia Álvarez Aguirre

Correo: Alicia.alvarez@ugto.mx

\section{Resumen}

Introducción: La sobrecarga del cuidador principal es un problema recurrente en personas que cuidan de adultos mayores con trastorno de Alzheimer. Objetivo: Determinar el nivel de sobrecarga del cuidador principal del adulto mayor con Enfermedad de Alzheimer. Metodología: Estudio descriptivo, participaron los cuidadores principales de cinco Centros Geriátricos de Querétaro, se aplicó la escala de Zarit ( $a=.86)$. En el análisis de datos se calculó estadística descriptiva y se contó con el consentimiento de las instituciones y de los participantes. Resultados: Participaron 22 cuidadores, del nivel de sobrecarga en los participantes se identificó nivel intenso en $77.3 \%$ y $4.5 \%$ leve. Conclusiones: La mayoría de los participantes se encontró en sobrecarga intenso, resultados que permiten diseñar e implementar estrategias para disminuir la sobrecarga de los cuidadores.

Palabras clave: Cuidadores, sobrecarga, Alzheimer.

\section{Abstract}

Introduction: Overload the primary caregiver is a recurring problem in caregivers of older adults with Alzheimer disorder. Objective: To determine the level of overload the primary caregiver of the elderly with Alzheimer's disease. Methodology: Descriptive study involved five primary caregivers Geriatric Centers Queretaro, Zarit scale was applied. In data analysis descriptive statistics were calculated and counted with the consent of the institutions and participants. Results: A total of 22 caregivers, overload level participants intense level was identified in $77.3 \%$ and $4.5 \%$ mild. Conclusions: Most of the participants found in intense overload, results that allow design and implement strategies to reduce the overload of caregivers.

Keywords: Caregivers, overload, Alzheimer. 


\section{INTRODUCCIÓN}

El proceso de envejecimiento trasciende en varios factores del desarrollo y el funcionamiento de las sociedades y también sobre el bienestar no sólo de los adultos mayores sino de los grupos más jóvenes. Algunos de los factores son la composición de la población activa y las modalidades de participación en la misma; los arreglos en cuanto a familia y hogar y las condiciones de salud de los Adultos Mayores (AM), entre otros [1].

El progresivo envejecimiento de las sociedades es debido fundamentalmente a la disminución de la tasa de la natalidad, al aumento de la esperanza de vida y a la disminución de la mortalidad, producto de la implementación de estrategias en materia de salud. Sin embargo, este comportamiento poblacional genera un incremento de la morbilidad crónica y degenerativa [2].

Existen en el mundo más de 416 millones de ancianos. Para el año 2025, los mayores de 60 años serán el 12\% de los habitantes. De éste total de ancianos, el $72 \%$ vivirá en los países en desarrollo, se espera que la esperanza de vida al nacimiento en Latinoamérica, se incremente de los 64.1 años que se tuvieron durante 1985, a 71.8 años para el año 2025 [1].

En México la población de 60 años y más asciende a 10055 379 personas, es decir el $9.06 \%$ del total de la población del país [3], este dato está cercano a los criterios establecidos por las Naciones Unidas: una población puede considerarse envejecida si más del $10 \%$ tiene 60 años o más [4]. En Querétaro la población de 60 años y más es de 136381 personas [3].

Entre el 2000 y 2010 en México, la población de adultos mayores creció a una tasa anual de $3.8 \%$; se espera que esta población se duplique en 18.4 años. En este grupo existe una relación de 87 hombres por cada 100 mujeres y 9.7 personas dependientes por vejez por cada 100 en edad reproductiva. Por el lugar de residencia el $74 \%$ reside en localidades urbanas y $26 \%$ viven en el área rural. En cuanto a escolaridad $74.4 \%$ saben leer y escribir un recado y respecto al estado civil el $6.3 \%$ de la población es soltera, $60 \%$ está casado o unido y $33.7 \%$ alguna vez estuvo casado o unido. Además existen $44.7 \%$ de los hogares donde el jefe de familia es una persona de 60 años y más, estas familias son de tipo nuclear [3].

Por otra parte los estados con mayor proporción de adultos mayores son Oaxaca (10.7\%), Distrito Federal (11.3\%) y Veracruz (10.4\%). Y las entidades con menor proporción son Baja California Norte (6.8\%), Baja California Sur (6.6\%) y Quintana Roo (4.8\%). Para Querétaro la proporción es de $7.46 \%$ [3].

En el contexto europeo y mexicano, la gran mayoría de las personas mayores prefieren envejecer en casa, en su entorno familiar, manteniendo

su

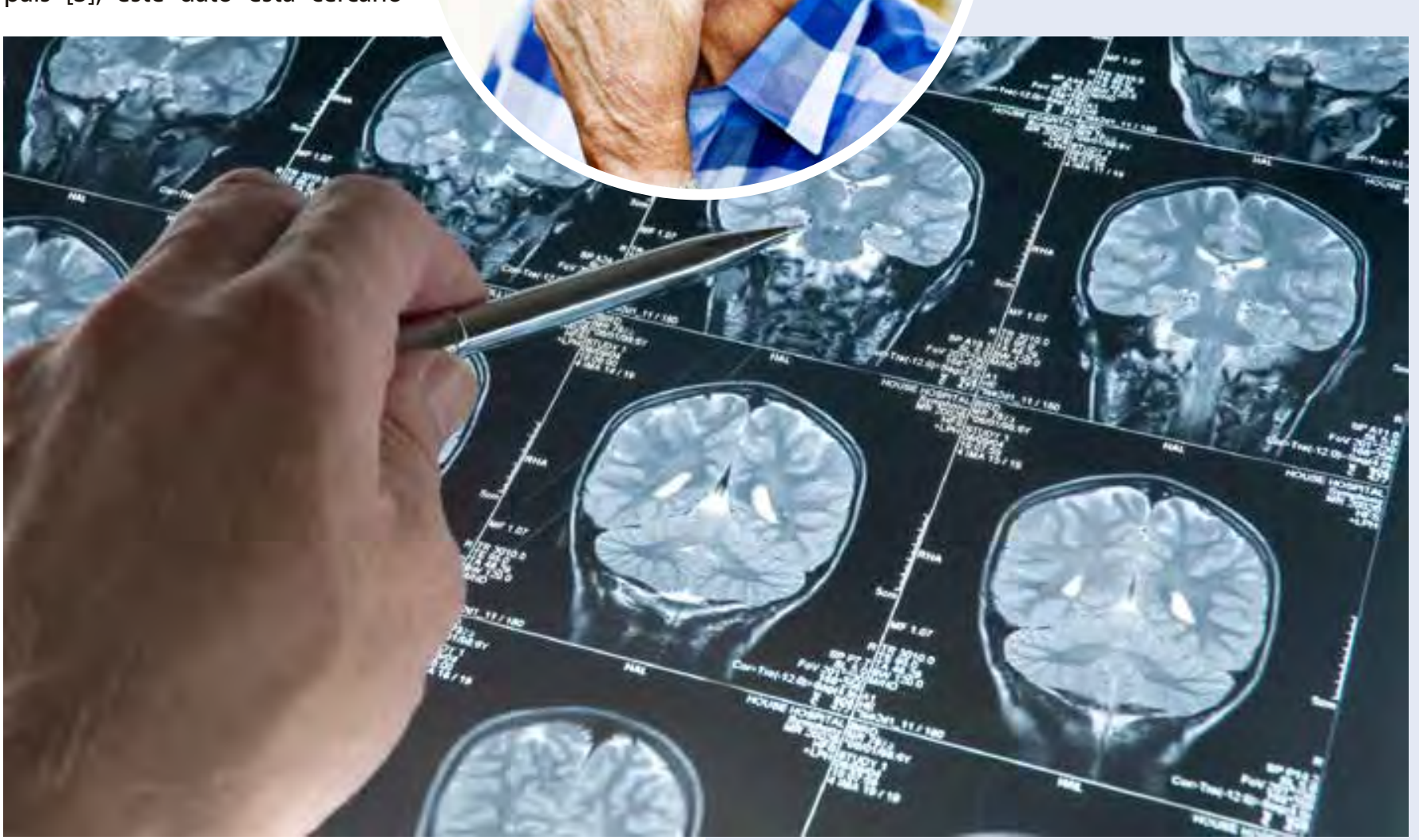




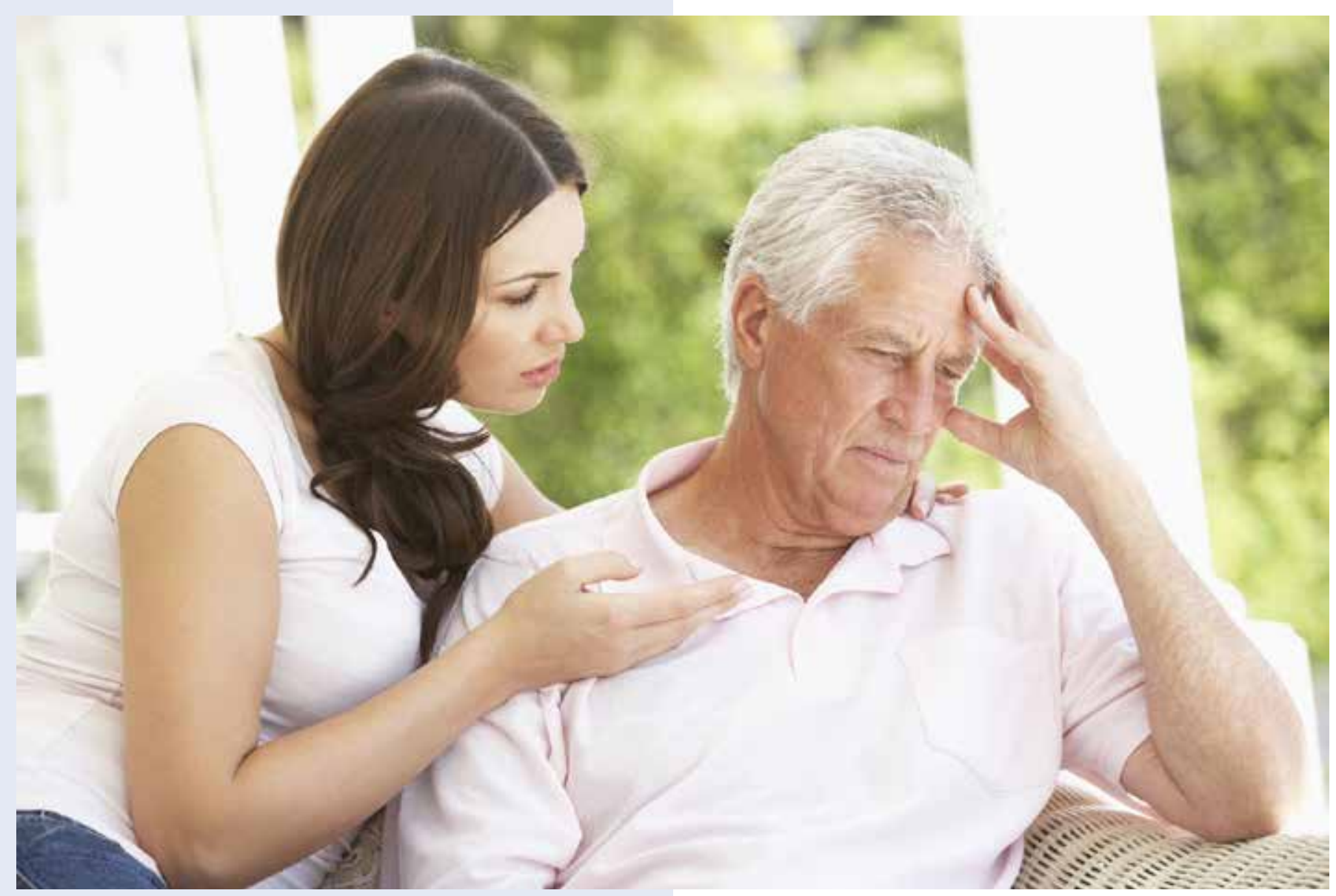

independencia y evitando así, en la gran mayoría de los casos, la soledad y el desarraigo familiar y social [2]. En este sentido el número de personas de este grupo de edad que requieren cuidados está aumentando, cuidados que son provistos por su familia [5].

Una de las enfermedades que más deterioro funcional y por tanto, mayor estrés proporcionan al cuidador, es la demencia. Esta es una enfermedad del sistema nervioso central, dada por alteraciones de los procesos mentales superiores, que modifica la personalidad y la conducta de las personas que la padecen. La más común es la Enfermedad Alzheimer (EA), que se atribuye a más de 70\% de los casos con dicha enfermedad [6]. En el mundo cerca de 35.6 millones de personas viven con demencia; se espera que para el 2030 este número se duplique (65.37 millones) y para el 2050 se triplique (115.4 millones) [6]. En los resultados de la Encuesta Nacional sobre Envejecimiento en México [7], se encontró una prevalencia nacional de Alzheimer de $6.1 \%$ para mayores de 60 años con una incidencia de 27.3 por cada mil adultos mayores por año, y en Querétaro, 804 personas cursan con esta enfermedad.

El anciano con Alzheimer tiene poca capacidad de adaptarse a su situación y, por lo tanto, son los que lo rodean quienes constantemente han de adaptarse a las necesidades creadas. A medida que la demencia va progresando, el enfermo se vuelve más inseguro de lo que le rodea y lo que los demás quieren de él, muchos pacientes se refugian en las personas que transmiten mejor el sentimiento de seguridad [8].

Los cuidadores principales de personas con demencia asumen las principales tareas y responsabilidades de cuidar. En la mayoría de los casos se trata de familiares directos o amigos muy próximos. Generalmente son las esposas o hijas de edad comprendida entre los 45 y los 70 años, que conviven con el adulto mayor, o bien le dedican una parte importante de su tiempo. Lo anterior compromete la salud mental social y física del cuidador [2].

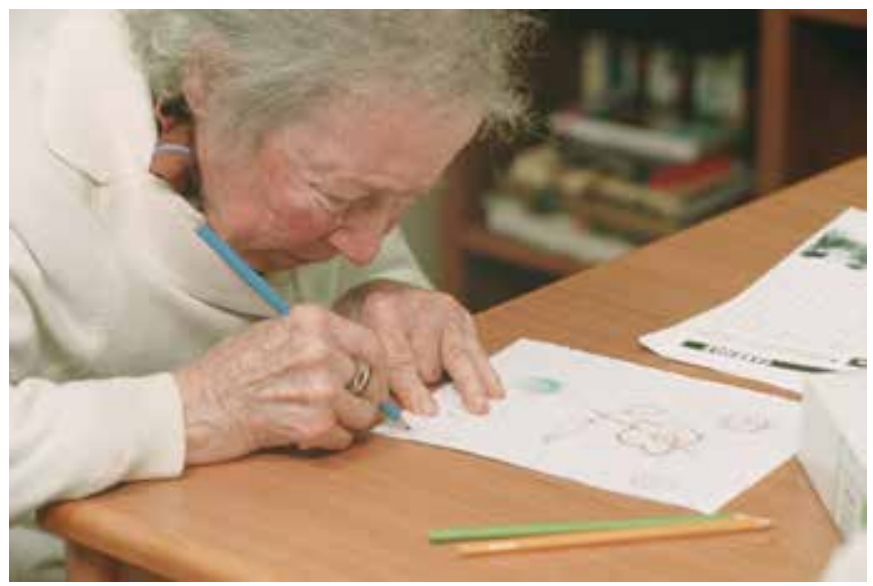




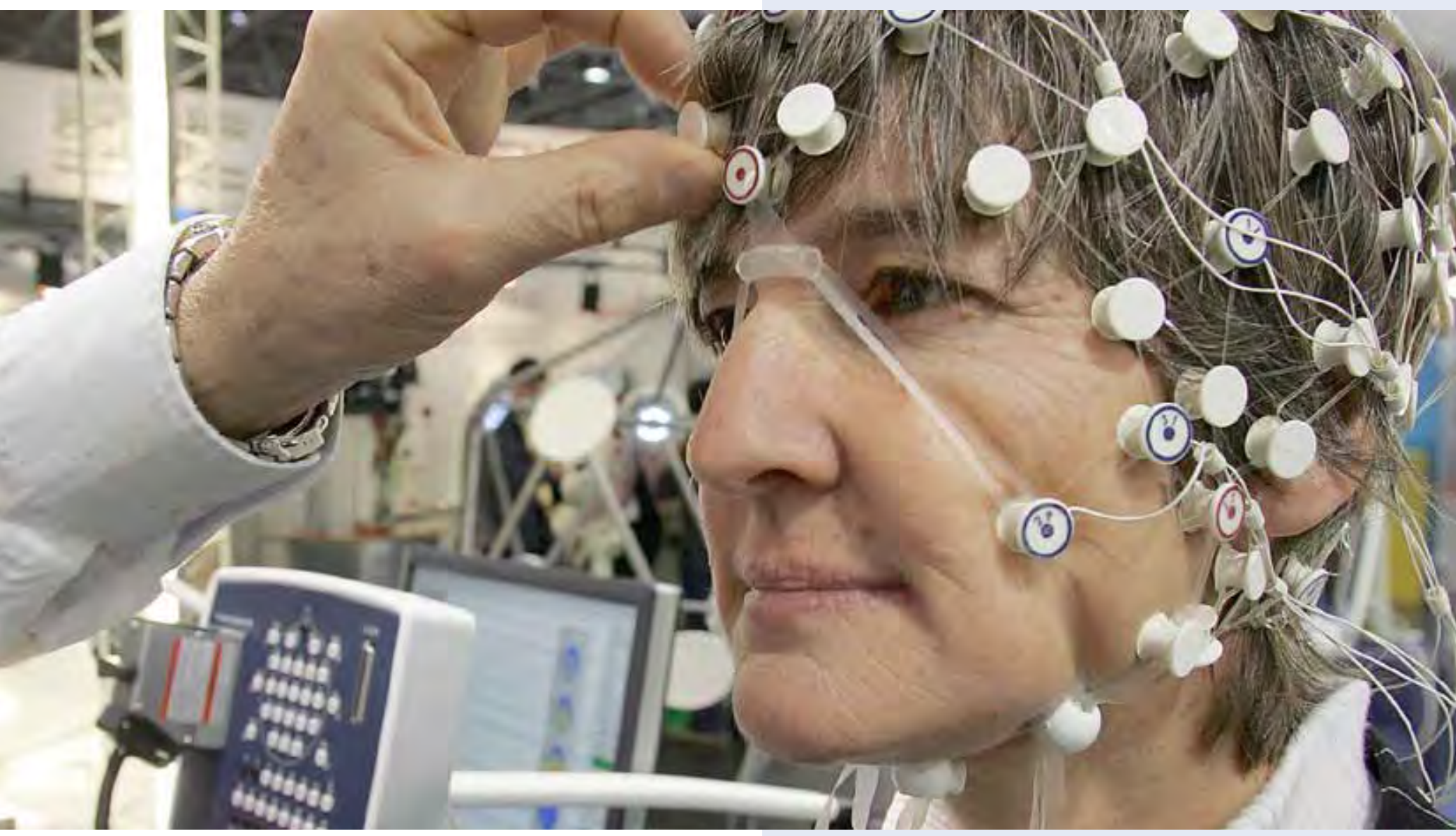

En este sentido, los cuidadores de adultos mayores con EA, se afectan en su bienestar físico, emocional y socioeconómico, lo que puede conllevar a que presenten estrés, ansiedad, alteraciones en sus relaciones laborales, entre otras consecuencias derivadas del cuidado de las personas con EA $[9,10]$. La afección que presentan los cuidadores y el sentimiento de que su vida ha cambiado desfavorablemente a partir de cuidar se le conoce como carga, término estudiado por Zarit [11], quien define la sobrecarga del cuidador como "la manera en qué y cómo los cuidadores perciben la salud tanto física como mental,

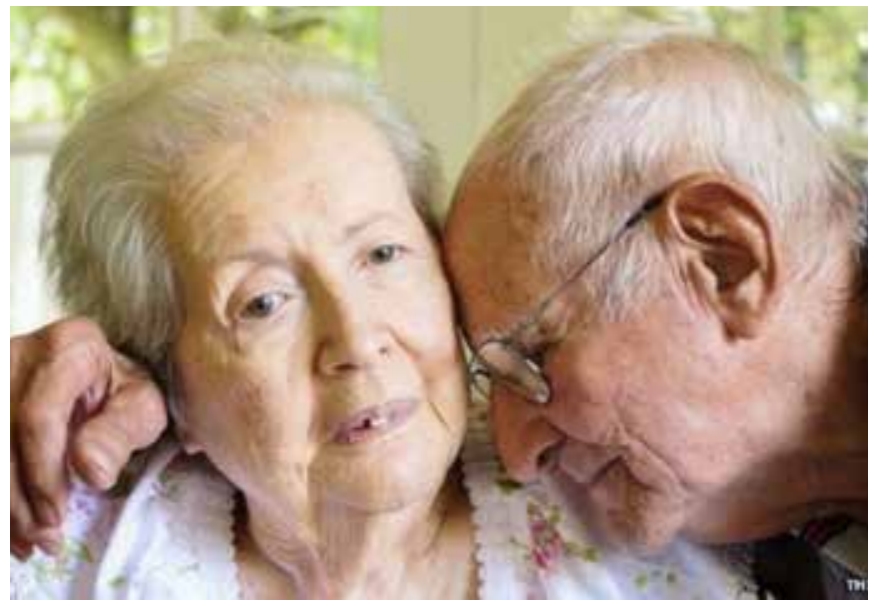

estado financiero y vida social como resultado del cuidado que ejerce sobre los pacientes".

La sobrecarga en el cuidador principal puede ser expresada por problemas físicos, como cefalea, astenia, fatiga crónica, alteración del ciclo del sueño, así como problemas psíquicos manifestados en depresión y ansiedad [12]. Por lo que una mayor sobrecarga deteriora la salud mental social y física del cuidador, aunado a que el cuidador no busca ayuda de los profesionales de salud postergándose y priorizando los problemas de su familiar dependiente, situación que se compromete aún más con un adulto mayor con EA.

Por lo anterior el objetivo del estudio fue determinar el nivel de sobrecarga del cuidador principal del adulto mayor con Enfermedad de Alzheimer. La relevancia de abordar este tema, es que los resultados permitirán proponer estrategias dirigidas a la prevención de la sobrecarga del cuidador principal y en un futuro ser la base para la planeación de intervenciones basadas en evidencia empírica.

\section{METODO}

Estudio descriptivo [13], que contó con la participación de 22 cuidadores procedentes de cinco Centros Geriátricos de Querétaro, México, seleccionados por conveniencia.

Para recabar la información sociodemográfica se diseñó 


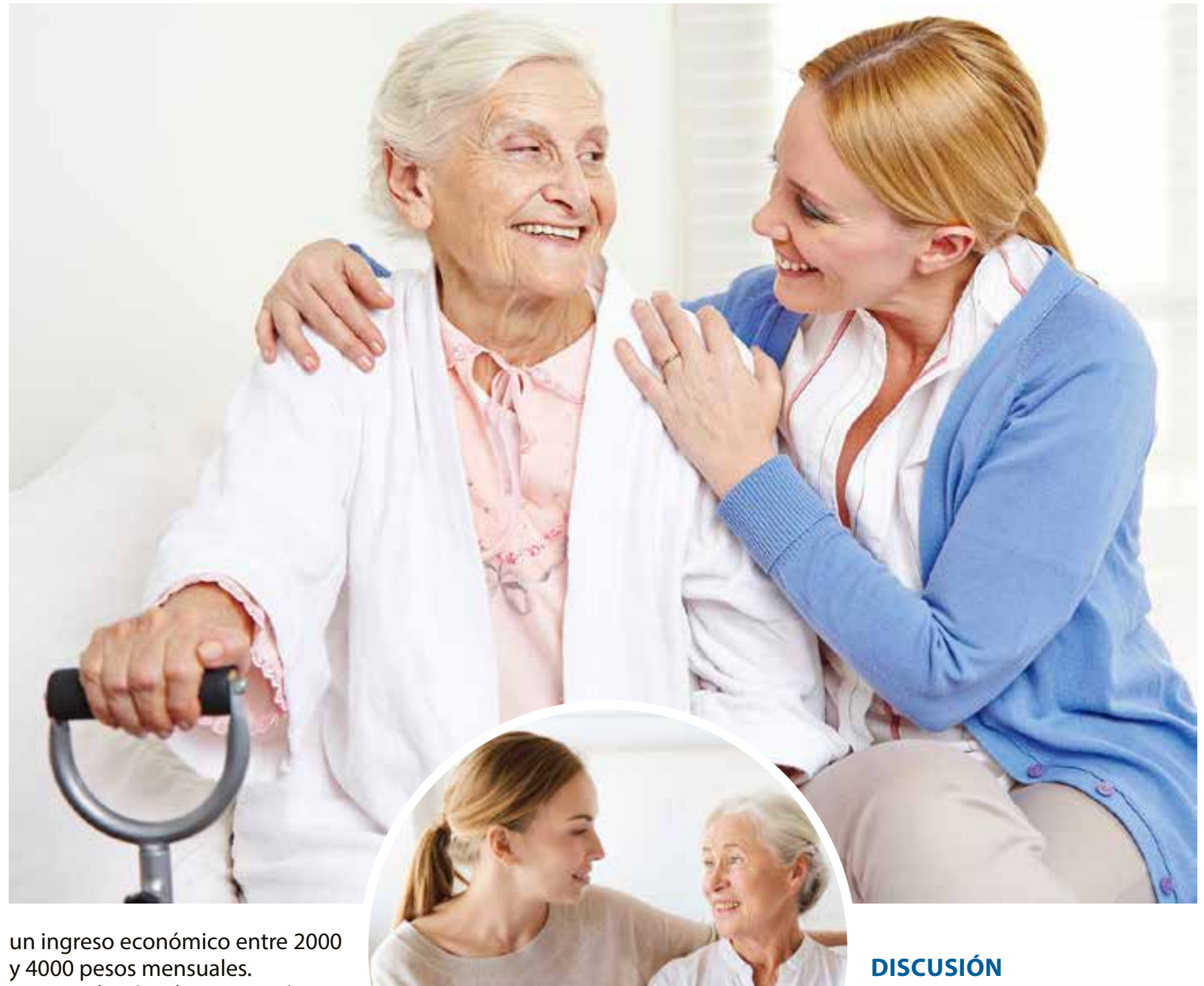

En relación a las características de sobrecarga de los participantes se destaca que una de cada diez, casi siempre se siente indeciso sobre qué hacer con su familiar, cree que su familiar espera que sea la persona que lo cuide como si fuera la única persona de quien depende, cree que no tiene suficiente dinero para cuidar a su familiar. Además, dos de cada diez casi siempre desearían dejar el cuidado de su familiar a otro y creen que serían incapaces de cuidarlo por más tiempo, sienten que han perdido el control de su vida desde la enfermedad de su familiar y creen que debería hacer más por su familiar. Asimismo, cuatro de cada diez casi siempre experimentan un grado de carga por el hecho de cuidar a su familiar.

Respecto al nivel de sobrecarga del cuidador principal se encontró sobrecarga intensa en el $77.3 \%$ de los participantes, sobrecarga leve en el $4.5 \%$ y sin sobrecarga en el $18.2 \%$.
El presente estudio permitió verificar el objetivo propuesto: determinar el nivel de sobrecarga del cuidador principal del adulto mayor con Enfermedad de Alzheimer, donde los resultados mostraron que la mayoría de los participantes se encontraron en sobrecarga intensa resultado similar a lo referido por Flores, Rivas y Seguel [10], quienes reportaron que seis de cada diez cuidadores se encontraban en ese nivel.

Además, de contar con evidencia empírica sobre el fenómeno de estudio, que permitirá proponer estrategias de seguimiento en esta población en específico. Por ejemplo implementar actividades de manejo de emociones, actividad física, ocupacional, entre otras que permitan al cuidador principal promover su salud mental. Mismas que podrían ser implementadas por equipos multidisciplinarios en los centros geriátricos. 


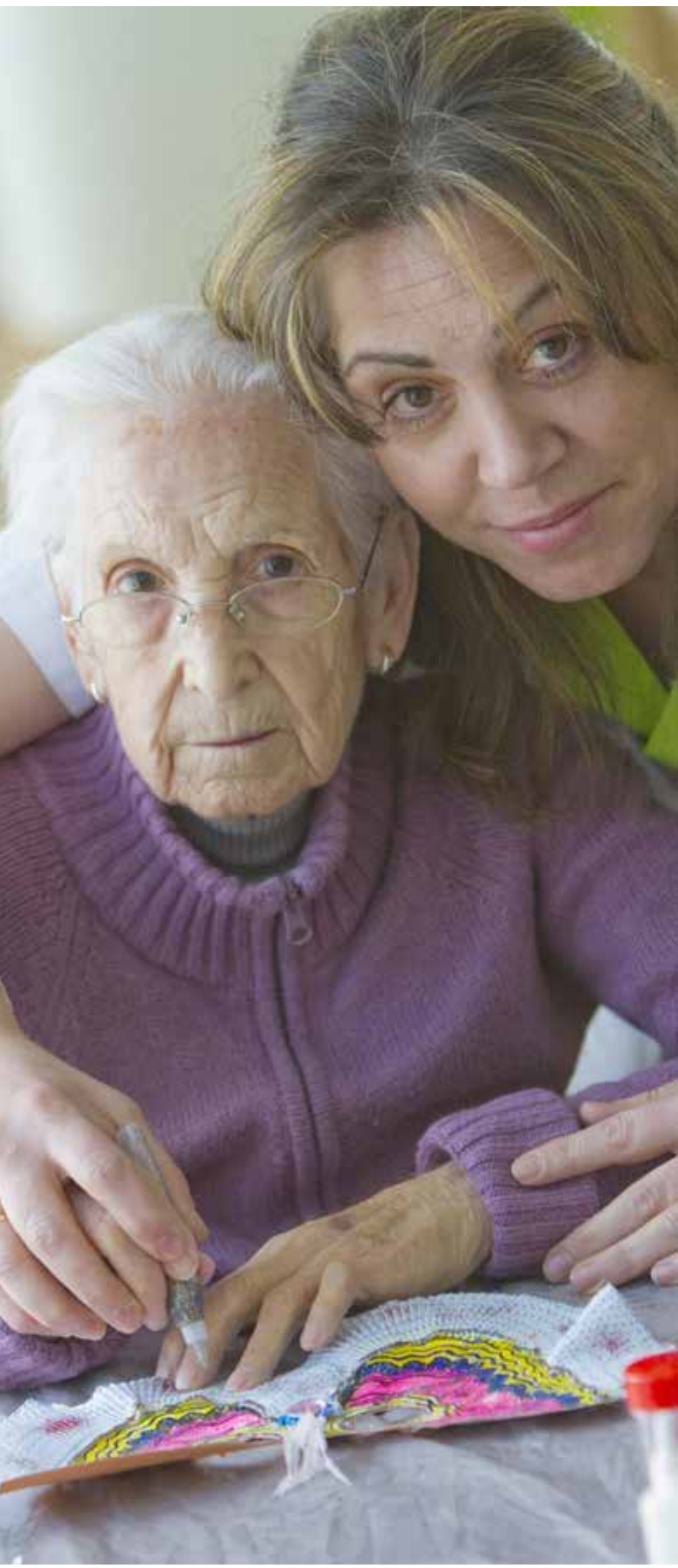

\section{CONCLUSIÓN}

Con base en los resultados que se obtuvieron en el presente estudio, se considera que se debe incluir dentro de las instituciones geriátricas una estrategia enfocada hacia el cuidador y el fortalecimiento de una red de apoyo social, tanto en el seno de la familia como alrededor de ellas, realizando un esfuerzo para la concientización de la familia y del propio cuidador en la necesidad de poder crear un soporte familiar para su beneficio. Además, programas de intervención de apoyo social para los cuidadores principales, orientados hacia el manejo de habilidades emocionales e instrumentales que permitan una mayor capacidad para afrontar los problemas que con el tiempo aparezca.

\section{BIBLIOGRÁFÍA}

[1] Secretaria de Salud, Programa de acción específico 20072012: Envejecimiento. México: Secretaria de salud, 2008.

[2] M. Ballester, L. Borrás, G. Corbalán, B. Hernandez, G. Paul, J. Pérez, "Perfil de salud y diagnósticos de enfermería en la población de atención domiciliaria del Área Básica de Salud de Castellar del Vallés", NUREInv., vol 5, no32, pp. 1-18, 2008.

[3] Instituto Nacional de Estadística y Geografía, Censo de población y vivienda 2010. México: INEGI, 2012.

[4] Organización de las Naciones Unidas, Envejecimiento de la población mundial. New York: ONU, 2009.

[5] Y. Yamamoto, Y. Hayashino, T. Higashi, M. Matsui, S. Yamazaki, M. Takegami, Y. Miyachi, S. Fukuhara, "Keeping vulnerable elderly patients free frompressure ulcer is associated with high caregiver burden in informal caregivers", Journal of evaluation in clinical practice, vol.16, pp. 585-589, 2008.

[6] Organización Mundial de la Salud. (2016) Demencia. Acceso Oct 2016. [en línea]. Disponible: http://www.who.int/ mediacentre/factsheets/fs362/es/

[7] Instituto Nacional de Estadística y Geografía, Encuesta Nacional sobre Salud y Envejecimiento en México 2012. México: INEGI, 2012.

[8] M. Pérez, J. Llibrel, “Características sociodemográficas y nivel de sobrecarga de cuidadores de ancianos con enfermedad de Alzheimer", Revista Cubana de Enfermería, vol. 26, no. 3, pp. 104-116, 2010.

[9] A. Espin, "Factores de riesgo de carga en cuidadores informales de adultos mayores con demencia", Revista Cubana de Salud Pública, vol. 38, no. 3, pp. 393-402, 2010.

[10] G. E. Flores, R. E. Rivas, P. F. Seguel, “Nivel de sobrecarga en el desempeño del rol del cuidador familiar de adulto mayor con dependencia severa", Ciencia y enfermería, vol. 18, no. 1, pp. 29-41, 2012.

[11] S. Zarit, K. Reever, J. Bach-Peterson, "Relatives of the impaired elderly: correlates of feelings of burden", Gerentologyst, vol 20, no. 6, pp.649-655, 1980

[12] A. Martins, T. Pereira, L. Silva, L. Correa, J. Ferreira, R. Partezani, "La sobrecarga y desánimo en los cuidadores de adultos mayors", Florianapolis, vol. 21, no. 2, pp.304-12, 2012.

[13] E. Pineda, E. Alvarado, F. Canales, Metodología de la investigación. Washington: OPS, 2008.

[14] Secretaria de Salud. (2014) Reglamento de la Ley General de Salud en Materia de Investigación para la Salud. Acceso Abr 2015. [en línea]. Disponible: http://www.diputados.gob.mx/ LeyesBiblio/regley/Reg_LGS_MIS_ref01_02abr14.doc

[15] D. Polit, B. Hungler, Investigación científica en ciencias de la salud, México: McGraw-Hil, 1999. 